\title{
NEW RESULTS ON SOME CLASSICAL PARABOLIC FREE-BOUNDARY PROBLEMS
}

\author{
BY \\ A. FASANO AND M. PRIMICERIO \\ Istituto Matematico, Università di Firenze \\ With an appendix by A. LACEY (New College, Oxford)
}

1. Introduction. In [1] we analyzed a class of free-boundary problems for the heat equation in one space dimension, releasing the sign restrictions on the data and the latent heat usually required in the Stefan problem. Problems of this kind have been studied by other authors also in connection with the freezing of a supercooled liquid and with decision theory (see e.g. [2]-[16], and the references quoted herein).

Two major problems remain open or not completely solved, namely (i) does any solution exist when the datum prescribed on the free boundary $x=s(t)$ does not fit the initial datum at $x=s(0)$ ?; (ii) how are the data related to the possibility of continuing the solution in arbitrarily large time intervals?

Sec. 2 of this paper contributes toward answering the above questions. Special results which are scattered in the literature cited can be found in the framework of our analysis (sometimes with relevant simplifications of the arguments).

It is known that some free-boundary problems with the Cauchy data prescribed on the free boundary can be reduced to schemes of the type mentioned above, provided that suitable compatibility conditions are fulfilled by the data. A typical example in which such conditions are violated is given by the diffusion-consumption of oxygen in insulated living tissues, when the initial oxygen distribution coincides with the steady-state profile corresponding to a given constant input (see [14] and [17]-[23]).

This case is considered in Sec. 3, there we prove the existence of a smooth solution and remark that the associated problem for the time derivative of the oxygen concentration is of the type considered in Sec. 2 but with an initial datum behaving like a " $\delta$ function" at the origin. A very sharp estimate of the lifetime of the tissue is also obtained by means of elementary calculations.

In Sec. 4 we prove some comparison theorems for the solutions of the problems dealt with in the preceding sections.

As an interesting consequence, a nonexistence theorem will identify a class of initial data such that the answer to question (i) above is negative.

2. Some special topics in Stefan problems of general type. Let us consider the following problem: find a triple $T, s(t), z(x, t)$ such that:

* Received March 1, 1979; revised version received February 26, 1980. 
(i) $T>0$;

(ii) $s(t)$ is a positive continuous function in $[0, T), s(t) \in C_{1}(0, T)$;

(iii) $z(x, t)$ is a bounded function continuous in $0 \leq x \leq s(t), 0 \leq t<T$ with the possible exception of the point $(1,0)$ and such that $z_{x}$ is continuous for $0 \leq x \leq$ $s(t), 0<T, z_{x x}$ and $z_{t}$ are continuous for $0<x<s(t), 0<t<T$;

(iv) the following conditions are satisfied:

$$
\begin{aligned}
z_{x x}-z_{t} & =0, & & D_{T}=\{(x, t): 0<x<s(t), 0<t<T\} ; \\
s(0) & =1 ; & & \\
z(x, 0) & =h(x), & & 0<x<1 \\
z_{x}(0, t) & =0, & & 0<t<T ; \\
z(s(t), t) & =0, & & 0<t<T ; \\
z_{x}(s(t), t) & =-\dot{s}(t), & & 0<t<T .
\end{aligned}
$$

Here $h(x)$ is a given continuous function in $[0,1]$; since it is not prescribed to be positive, the problem differs substantially from the ordinary Stefan problem (for which the existence for any $T$ is proved and $\dot{s}(t)>0)$. Nevertheless, if $h(1)=0$ and $h(x)$ is Hölder-continuous for $x=1$ this problem possesses one solution for suitable $T$ "sufficiently small" (see [1], where uniqueness and continuous dependence are also discussed, and [9], where it is proved that the free boundary is analytic in $(0, T)$ ). Moreover, if a solution exists, then three cases can occur (see [1, I] Theorem 8):

(A) The problem has a solution with arbitrarily large $T$;

(B) There exists a constant $T_{0}>0$ such that: $\lim _{t \rightarrow T_{0-}} s(t)=0^{1}$;

(C) There exists a constant $T_{1}>0$ such that

$$
\inf _{t \in\left(0, T_{1}\right)} s(t)>0, \quad \lim _{t \rightarrow T_{1-}} \inf \dot{s}(t)=-\infty .
$$

We shall investigate the occurrence of these cases in connection with the behavior of the initial datum $h(x)$.

A first simple result is Lemma 2.1 below. Let us define

$$
\begin{aligned}
Q & =1+\int_{0}^{1} h(x) d x, \\
M_{1} & =-\int_{0}^{1} x h(x) d x, \\
M_{2} & =-\int_{0}^{1} x^{2} h(x) d x
\end{aligned}
$$

and prove

LEMMA 2.1. If $T, s(t), z(x, t)$ solve (i)-(iv), then

$$
s(t)=Q-\int_{0}^{s(t)} z(x, t) d x, \quad t \in(0, T)
$$

\footnotetext{
${ }^{1}$ This limit exists since otherwise for any $\bar{t}$ in $\left(0, T_{0}\right), z(x, \bar{t})$ would have infinitely many zeros.
} 


$$
\begin{array}{ll}
{\left[s^{2}(t)-1\right] / 2=-M_{1}+\int_{0}^{t} z(0, \tau) d \tau-\int_{0}^{s(t)} x z(x, t) d x,} & t \in(0, T), \\
{\left[s^{3}(t)-1\right] / 3=-M_{2}+2 \int_{0}^{t} \tau \dot{s}(\tau) d \tau-\int_{0}^{s(t)} x^{2} z(x, t) d x,} & t \in(0, T) .
\end{array}
$$

Proof. Consider Green's identity

$$
\iint_{D_{t}}\left(v \mathbf{L} u-u \mathbf{L}^{*} v\right) d x d \tau=\oint_{\partial D_{t}}\left[\left(v u_{x}-u v_{x}\right) d \tau+u v d x\right],
$$

where $\mathbf{L}$ denotes the heat operator and $\mathbf{L}^{*}$ its adjoint. Formulas (2.10), (2.11), (2.12) are obtained by setting $u=z(x, t)$ and $v=1, v=x, v=x^{2}-2 t$ respectively.

Other relationships of the same kind could be obtained using higher-order polynomials for $v(x, t)$.

As a consequence of $(2.10)$ we can prove that for some choice of the function $h(x)$ no solution to (i)-(iv) can exist.

THEOREM 2.2 (nonexistence). If $h(x)$ is a constant not exceeding - 1 problem (i)-(iv) has no solution.

Proof. Under the above assumption $h(x) \equiv-1+Q$ with $Q \leq 0$. Should (i)-(iv) have any solution $T, s, z$, it would be

$$
\begin{array}{ll}
0<s(t)<1, & t \in(0, T), \\
z(x, t)>-1+Q, & \text { in } D_{T},
\end{array}
$$

because of $(2.1)-(2.6)$ and of the maximum principle. Thus from $(2.10)$ we would have

$$
s(t)<Q+(1-Q) s(t), \quad t \in(0, T),
$$

i.e. $0<Q[1-s(t)]$, which is a contradiction to $Q \leq 0, s<1$.

Remark 2.3. Owing to the above result it will be tacitly understood that $h \not \equiv-1+Q$ with $Q \leq 0$.

Our next aim will be to look for some conditions on $h(x)$ giving an a priori characterization of cases (A), (B), (C).

Lemma 2.4. Assume $h(x)$ satisfies

(H1) there exists a positive constant $H$ such that

$$
h(x) \geq-H(1-x)
$$

and let $T, s, z$ be a solution of (i)-(iv) such that

$$
s_{T} \equiv \inf _{t \in(0, T)} s(t)>0 .
$$

If there exist two constants $d \in\left(0, s_{T}\right), z_{0} \in(0,1)$ such that $H d<z_{0} \ln 2$ and

$$
z(s(t)-d, t)>-z_{0}, \quad t \in(0, T),
$$

then

$$
\dot{s}(t) \geq \min \left\{-H / z_{0}, d^{-1} \ln \left(1-z_{0}\right)\right\} .
$$


Proof. For any $\varepsilon \in(0, T)$ consider the domain

$$
\Omega_{\varepsilon}=\{(x, t): s(t)-d<x<s(t), \quad 0<t<T-\varepsilon\}
$$

and the function

$$
w(x, t)=-z_{0}\left(1-e^{-a d}\right)^{-1}(1-\exp [a(x-s(t))]),
$$

where $a$ is a positive constant to be determined.

We have

$$
\begin{aligned}
w_{x x}-w_{t} & =z_{0}\left(1-e^{-a d}\right)^{-1}\left(a^{2}+a \dot{s}\right) \exp [a(x-s(t))], & & \text { in } \Omega_{\varepsilon} \\
w(x, 0) & =-z_{0}\left(1-e^{-a d}\right)^{-1}(1-\exp [a(x-1)]), & & 1-d<x<1, \\
w(s(t), t) & =0, & & 0<t<T-\varepsilon, \\
w(s(t)-d, t) & =-z_{0}, & & 0<t<T-\varepsilon .
\end{aligned}
$$

Choose the constant $a$ such that

$$
a \geq H / z_{0} ;
$$

this implies that $w_{x}(1-d, 0) \geq H$ and consequently (since $w<0, w_{x x}>0$ and $(2.13)$ holds) $w(x, 0) \leq h(x)$ for $x \in[1-d, 1]$. Besides (2.17), we shall require that the constant $a$ satisfy the following inequality:

$$
a \geq-\sigma_{\varepsilon}, \quad \sigma_{\varepsilon} \equiv \inf _{t \in(0, T-\varepsilon)} \dot{s}(t),
$$

so that

$$
w_{x x}-w_{t} \geq 0
$$

Since (2.13) and (2.8) yield $w(s(t)-d, t) \leq z(s(t)-d, t)$, we have from the maximum principle

$$
z(x, t) \geq w(x, t) \text { in } \Omega_{\varepsilon}
$$

and, recalling (2.12),

$$
z_{x}(s(t), t) \leq a z_{0}\left(1-e^{-a d}\right)^{-1}, \quad 0<t<T-\varepsilon .
$$

There are two possibilities: either $-\sigma_{\varepsilon} \leq H / z_{0}$ for any $\varepsilon \in(0, T)$ (and then (2.16) follows letting $\varepsilon \rightarrow 0)$ or $-\sigma_{\varepsilon}>H / z_{0}$ for some $\varepsilon \in(0, T)$. In the latter case choose $a=-\sigma_{\varepsilon}$ and obtain from (2.6) and (2.18)

$$
\sigma_{\varepsilon} \geq \sigma_{\varepsilon} z_{0}\left[1-\exp \left(\sigma_{\varepsilon} d\right)\right]^{-1}
$$

whence $\left(\sigma_{\varepsilon}\right.$ is negative)

$$
\sigma_{\varepsilon} \geq d^{-1} \ln \left(1-z_{0}\right)
$$

and the conclusion of the proof of the lemma.

Next, we have the following lemma.

Lemma 2.5. Assume $h(x)$ satisfies

(H2) the equation $h(x)=-1$ has at most one root in $[0,1]$; if $h(1)<-1$, it has no root in $[0,1]$, 
and let $T, s(t), z(x, t)$ solve (i)-(iv). Then

$$
Q \leq 0 \Rightarrow z(0, t)<-1,
$$

(II) $Q \geq 0 \Rightarrow$ either there are no points in $D_{T}$ where $z(x, t)=-1$, or the level curve $z=-1$ is separated by a positive distance from the free boundary $x=s(t)$, for $t$ in $(0, T]$ and such that $s(t)>0$.

Proof. First, consider the case $h(x) \geq-1$, which implies $Q \geq 0$ (actually, $Q>0$ because of $(\mathrm{H} 2))$. By the maximum principle, $z(x, t)>-1$ in $D_{T}$ and (II) is satisfied. In the other cases, consider the level curve $z(x, t)=-1$ originating from $t=0$ (which is unique because of $(\mathrm{H} 2))$ and note that if $z(\hat{x}, \hat{t})=-1$, then $z(x, \hat{t})<-1$ for $x<\hat{x}, z(x, \hat{t})>-1$ for $x>\hat{x}$. Now, from (2.10) it is easily seen that if $Q=0,0<\hat{x}<s(\hat{t})$; if $Q<0$, then $\hat{x}>0$; if $Q>0$, then $\hat{x}<s(t)$.

Remark 2.6. Assumption (H2) can be weakened in the following sense:

$\left(\mathrm{H} 2^{\prime}\right)$ Either $h(x) \leq-1$ in $[0,1]$ or no point $x^{*} \in(0,1)$ such that $h\left(x^{*}\right)<-1[>-1]$ belongs to an interval $\left(x_{1}, x_{2}\right) \subset(0,1)$ such that $h\left(x_{i}\right) \geq-1[\leq-1]$ for $i=1,2$ and that $h\left(x_{1}\right) h\left(x_{2}\right)<1[>1]$. Moreover, if $h(1)<-1$ then $h(x) \leq-1$.

Indeed, the proof of the lemma needs only minor changes (recall also that $h \equiv-1$ is excluded by Remark 2.3 ).

Remark 2.7. When in $(\mathrm{H} 2)$ or $\left(\mathrm{H} 2^{\prime}\right)$ the value -1 is replaced by the value $-1+Q$, we have that

(III) if $s \leq 1$ in $(0, T) Q \geq 0 \Rightarrow$ level curve $z=-1+Q$ separated from $s(t)$,

$$
Q \leq 0 \Rightarrow z(0, t)<-1+Q ;
$$

(IV) if $s \geq 1$ in $(0, T) Q \geq 0 \Rightarrow z(0, t)<-1+Q$,

$$
\begin{gathered}
Q \leq 0 \Rightarrow \text { level curve } z=-1+Q \\
\text { separated from } s(t) .
\end{gathered}
$$

Note that $(\mathrm{H} 2)\left[\left(\mathrm{H}^{\prime}\right)\right]$ is clearly satisfied by monotone increasing (nondecreasing) functions (for which (III) and (IV) hold as well). The monotonicity of the function $h(x)$ will be used in the sequel (see Proposition 2.11, Theorem 2.13) to improve some estimates obtained under $\left(\mathrm{H} 2^{\prime}\right)$.

The next lemma gives a priori estimates for the functions $s(t), z(x, t)$.

Let

$$
S_{0}=1+\int_{0}^{1} h^{+}(x) d x,
$$

where $h^{+}(x)=\max [0, h(x)]$, and let $Z(x, t)$ be the solution of the heat equation in the half-strip $0<x<S_{0}, t>0$ with the initial condition

$$
\begin{aligned}
& Z(x, 0)=|h(x)|, \quad 0<x<1, \\
& =0, \quad 1 \leq x<S_{0} \quad \text { (if } S_{0}>1 \text { ), }
\end{aligned}
$$

and the boundary conditions

$$
Z_{x}(0, t)=0, \quad Z\left(S_{0}, t\right)=0, \quad t>0 .
$$


Then we have

LemMa 2.8. For any solution $(T, s, z)$ of (i)-(iv) it is true that

$$
\begin{gathered}
s(t) \leq S_{0}, \quad \forall t \in(0, T), \\
|z(x, t)| \leq Z(x, t) \quad \text { in } D_{T} .
\end{gathered}
$$

Proof. Let $\left(S_{n}(t), Z_{n}(x, t)\right)$ be the solution of the classical Stefan problem for the heat equation with the initial conditions

$$
\begin{aligned}
& S_{n}(0)=1+\frac{1}{n}, \quad Z_{n}(x, 0)=h^{+}(x), \quad 0<x<1, \\
& =0, \quad 1 \leq x<1+\frac{1}{n},
\end{aligned}
$$

$n=1,2, \ldots$.

If $(T, S, z)$ is a solution of (i)-(iv), the inequality $s(t)<S_{n}(t)$ follows from the monotone dependence theorem of $[1, I]$. Moreover, the equation

$$
S_{n}(t)=S_{0}+\frac{1}{n}-\int_{0}^{S_{n}(t)} Z_{n}(x, t) d x,
$$

and $Z_{n}(x, t) \geq 0$ imply $S_{n}(t) \leq S_{0}+1 / n$. This proves the first part of the lemma. The second part is an elementary consequence of the maximum principle.

At this point we are able to prove

TheOREM 2.9. Let (H1), (H2') be verified. Then for any solution of (i)-(iv)

$$
\begin{aligned}
& \text { (A) } \Leftrightarrow Q>0, \\
& \text { (B) } \Leftrightarrow Q=0, \\
& \text { (C) } \Leftrightarrow Q<0 .
\end{aligned}
$$

Proof of (2.20). $\Rightarrow$ : It suffices to perform the limit for $t \rightarrow T_{0}$ in $(2.10)$. $\Leftarrow$ : Recall $|z| \leq Z$ from Lemma 2.8 and note that $Z(x, t)$ tends to zero for $t \rightarrow+\infty$ uniformly with respect to $x \in\left[0, S_{0}\right]$. Now, since $Q=0$ implies $z(0, t)<-1$ because of Lemma 2.5 (I), case (A) is excluded. Moreover, Lemma 2.5 (II) and Lemma 2.4 imply that $\dot{s}(t)$ is bounded from below and also (C) is excluded.

Proof of (2.19). $\Rightarrow$ : Again from Lemma 2.8 we see that the right-hand side of (2.10) tends to $Q$ as $t \rightarrow+\infty$. Hence $s(t)$ has limit $s \rightarrow Q$ and thus $Q>0$ (since the case $Q=0$ was proved to imply (B)). $\Leftarrow$ : It suffices to exclude (C): this is immediately accomplished using Lemmas 2.4 and 2.5 .

Proof of (2.21). It follows immediately from (2.19) and (2.20).

There exist some relations between the occurrence of cases (A), (B), (C) and the quantities $Q, M_{1}, M_{2}$ not needing assumptions $(\mathrm{H} 1),\left(\mathrm{H}^{\prime}\right)$. More precisely, we prove the following

THEOREM 2.10. For any solution of (i)-(iv)

$$
\begin{aligned}
& \text { (A) } \Rightarrow Q \geq 0, \quad \lim _{t \rightarrow+\infty} s(t)=Q, \\
& \text { (B) } \Rightarrow Q=0, \\
& \text { (C) } \leftarrow Q<0 .
\end{aligned}
$$


Moreover, if $h \leq 0$ and a solution to (i)-(iv) exists,

$$
\begin{array}{lll}
\text { (A) } \Rightarrow M_{1}<\left(1-Q^{2}\right) / 2 \text { and } & M_{2}<\left(1-Q^{3}\right) / 3, \\
\text { (B) } \Rightarrow M_{1}<1 / 2 \text { and } & & M_{2}<1 / 3, \\
\text { (C) } \Leftarrow M_{1} \geq\left(1-Q^{2}\right) / 2 \text { or } & M_{2} \geq\left(1-Q^{3}\right) / 3 .
\end{array}
$$

Proof. The proof of (2.22), (2.23), (2.24) is accomplished in the same way as the corresponding statements in Theorem 2.9 .

To prove $\left(2.22^{\prime}\right),\left(2.23^{\prime}\right)$ it suffices to perform the limits (for $t \rightarrow+\infty, t \rightarrow T_{0}$ respectively) in (2.11), (2.12) and to note that $\dot{s} \leq 0$ because of the maximum principle. Finally, $\left(2.24^{\prime}\right)$ follows from $\left(2.22^{\prime}\right),\left(2.23^{\prime}\right)$.

An extension of $\left(2.24^{\prime}\right)$ is found in the Appendix.

In view of Theorem 2.10 , it is of some interest to consider a priori relationships among $M_{1}, M_{2}$ and $Q$. This is the purpose of the following proposition.

Proposition 2.11. If $h(x)$ is monotone nondecreasing,

$$
M_{1} \leq(1-Q) / 2, \quad M_{2} \leq(1-Q) / 3 ;
$$

if $h(x)$ is monotone nonincreasing,

$$
M_{1} \geq(1-Q) / 2, \quad M_{2} \geq(1-Q) / 3,
$$

the equality signs in (2.25), (2.26) holding if and only if $h$ is constant.

If $h(x)$ satisfies $\left(\mathrm{H} 2^{\prime}\right)$, then

$$
\begin{aligned}
& Q \geq 0 \Rightarrow M_{1}<1 / 2 \text { and } M_{2}<1 / 3 \text {, } \\
& Q<0 \Rightarrow M_{1}<1 / 2-Q \text { and } M_{2}<1 / 3-Q \text {. }
\end{aligned}
$$

Proof. Define

$$
H(x)=\int_{x}^{1} h(\xi) d \xi
$$

and note that

$$
\begin{aligned}
& M_{1}=-\int_{0}^{1} H(x) d x \\
& M_{2}=-2 \int_{0}^{1} x H(x) d x
\end{aligned}
$$

(it suffices to perform an integration by parts in (2.8), (2.9)).

Since $H(0)=-1+Q($ see $(2.7))$ and $H(1)=0$, if $h(x)$ is nondecreasing (then $H^{\prime}(x)$ is nonincreasing)

$$
H(x) \geq(1-Q)(x-1)
$$

and the converse is true if $h(x)$ is nonincreasing. The equals sign holds if and only if $h \equiv-1+Q$. Using the above results in (2.30), (2.31) proves the first part of the lemma.

Now let $h$ satisfy $\left(\mathrm{H}^{\prime}\right)$. This implies that the curve $y=H(x)$ cannot go below the straight lines $y=x-1$ (in the case $Q \geq 0$ ) or $y=x-1+Q$ (in the case $Q<0$ ). Then 
$H(x) \geq x-1$ (when $Q \geq 0$ ) and $H(x) \geq x-1+Q$ (when $Q<0$ ). Thus (2.27) and (2.28) follow from (2.30), (2.31).

Now, we prove two theorems giving a more precise insight into cases (B) and (C).

Let us define

$$
\bar{h}=\min _{[0,1]} h(x)
$$

and prove

THEOREM 2.12. For any solution of (i)-(iv) satisfying (B) it is true that $\bar{h}<-1$ and

$$
\begin{gathered}
\left.T_{0}>-(1 / 2)-M_{1}\right) / \bar{h} \\
\int_{0}^{T_{0}} s(t) d t=\left(1 / 3-M_{2}\right) / 2 ;
\end{gathered}
$$

if in addition ( $\left(\mathrm{H}^{\prime}\right)$ is satisfied, then

$$
\begin{gathered}
T_{0}<1 / 2-M_{1}, \\
\lim _{t \rightarrow T_{0-}} \inf \dot{s}(t)\left(T_{0}-t\right)^{1 / 2}=-\infty .
\end{gathered}
$$

Proof. First of all, recall (2.23) to get $Q=0$ and consequently $\bar{h}<-1$ (recall also Remark 2.3). To prove (2.34), pass to the limit for $t \rightarrow T_{0^{-}}$in (2.11) and note that $z(0, t)>\bar{h}$. The upper estimate (2.36) follows in the same way taking into account that $z(0, t)<-1$ (use (H2') to apply Lemma 2.5, (I) along with Remark 2.6).

Performing the same limit in (2.12) after an integration by parts of the term $\int_{0}^{t} \tau \dot{s}(\tau) d \tau$ yields $(2.35)$.

Finally, (2.37) is a consequence of the inequality $z(0, t)<-1$, which implies that $z(x, t)$ is discontinuous at $\left(0, T_{0}\right)$; indeed, the free boundary cannot lie below any parabola with vertex in $\left(0, T_{0}\right)$ (see e.g. [24]).

Recalling now (2.24), we state

Theorem 2.13. Assume $\left(\mathrm{H}^{\prime}\right)$. If $Q<0$, then $\bar{h} \leq-1+Q$ and, whenever a solution to (i)-(iv) exists,

$$
\inf _{\left[0, T_{1}\right]} s(t) \equiv s_{0}>Q /(1+h) .
$$

If in addition to $\left(\mathrm{H}^{\prime}\right)$ it is true that $h \leq 0$ and $Q \leq 0$, for any solution of (i)-(iv) satisfying (C) we have

$$
T_{1}<1 / 2-M_{1}-(1+\bar{h}) s_{0}^{2} / 2 .
$$

If $h(x)$ is nonpositive and nondecreasing, for any solution of (i)-(iv) satisfying (C) we have

$$
T_{1}<\left(1 / 2-M_{1}-s_{0} Q / 2\right) /\left(1-Q / s_{0}\right),
$$

irrespective of the value of $Q$.

Proof. From (2.10), (2.25) and the maximum principle we have

$$
s(t)-Q<-\bar{h} s(t), \quad \forall t \in\left(0, T_{1}\right),
$$


from which (2.38) follows (the inequality $\bar{h}<-1+Q<-1$ is obvious and implies $0<Q /(1+h)<1)$.

Assuming $h(x) \leq 0$, we have $\dot{s}(t) \leq 0$ and consequently

$$
s_{0}=\lim _{t \rightarrow T_{1-}} s(t) \text {. }
$$

By taking the limit $t \rightarrow T_{1}$ - in (2.11) and using $z(0, t)<-1$, which is valid even if $Q=0$ (Lemma $2.5,(\mathrm{I}))$, and $z(x, t)>\bar{h}$, inequality $(2.39)$ is easily derived.

Before proving (2.40), it is worth noting that this estimate of $T_{1}$ is sharper than (2.39): this follows from (2.38) and the assumption $Q \leq 0$ under which (2.39) has been proved.

Whenever $h(x)$ is nonpositive and nondecreasing, then (irrespective of the value of $Q$ ) we can assert that

$$
z_{x}(x, t)>0 \text { in } D_{T_{1}},
$$

owing to the maximum principle, and that

from (2.10).

$$
z(0, t)<-1+Q / s(t), \quad \forall t \in\left(0, T_{1}\right),
$$

Moreover, we can prove that

$$
-\int_{0}^{s(t)} x z(x, t) d x<s(t)[s(t)-Q] / 2 .
$$

This follows from integrating by parts the integral in (2.43):

$$
\int_{0}^{s(t)} x z(x, t) d x=[x \hat{z}(x, t)]_{0}^{s(t)}-\int_{0}^{s(t)} \hat{z}(x, t) d x,
$$

where

$$
\hat{z}(x, t)=\int_{0}^{x} z(\xi, t) d \xi ;
$$

noting that $\hat{z}(0, t)=0, \hat{z}(s(t), t)=Q-s(t)$ (from (2.18)), that $\hat{z}_{x}=z \leq 0$ (because of $h \leq 0)$ and that $\hat{z}_{x x}=z_{x}>0$ in $D_{T_{1}}($ see $(2.41))$, we arrive at the inequality

$$
\hat{z}(x, t)<(-1+Q / s) x \text { in } D_{T_{1}},
$$

from which $\int_{0}^{s(t)} \hat{z}(x, t) d x<-s(t)[s(t)-Q] / 2$, leading to (2.43).

Now, let $t \rightarrow T_{1}$ - in (2.11) and use (2.42), (2.43):

$$
\left(s_{0}^{2}-1\right) / 2<-M_{1}-T_{1}\left(1-Q / s_{0}\right)+s_{0}^{2} / 2-s_{0} Q / 2,
$$

from which (2.40) is obtained.

Remark 2.14. From (2.39) we get the a priori estimate

$$
T_{1}<-M_{1}-\bar{h} / 2 \text {. }
$$

Similarly, from (2.40) when $Q \leq 0$ we derive the a priori estimate

$$
T_{1}<\left(1 / 2-M_{1}-Q / 2\right) /(1-Q) \text {. }
$$

We notice that the right-hand sides of $\left(2.39^{\prime}\right)$ and of $\left(2.40^{\prime}\right)$ are positive as a consequence of the definitions of $M_{1}$, and $\bar{h}$ and of the inequality (2.25) (recall also Remark 2.3). 
However, our results do not guarantee a priori that the right-hand sides of (2.39) and (2.40) are positive: as a matter of fact, it is reasonable to conjecture that other cases of nonexistence can occur besides the one of Theorem 2.1.

For instance, if one proves that solutions depend monotonically on $h(x)$ and $s(0)$, then it could be proved that no solution can exist when $h(x)<-1$ in $(0,1)$. This analysis will be performed in Sec. 4 .

3. The oxygen diffusion-consumption problem. The following scheme:

$$
\begin{aligned}
u_{x x}-u_{t} & =1, & \text { in } & D_{T}=\{(x, t): 0<x<s(t), 0<t<T\}, \\
s(0) & =1, & & \\
u(x, 0) & =g(x), & & 0<x<1, \\
u_{x}(0, t) & =0, & & 0<t<T, \\
u(s(t), t) & =0, & & 0<t<T, \\
u_{x}(s(t), t) & =0, & & 0<t<T,
\end{aligned}
$$

is known as a mathematical model for the one-dimensional diffusion of oxygen in a living tissue [17]. In (3.1)-(3.6) $u$ denotes the oxygen concentration and the source term in (3.1) accounts for the oxygen consumption in the tissue.

In view of this application we shall assume that $g(x)$ is nonnegative and nonincreasing (and $g \not \equiv 0$ ) in order to have $u>0$ (indeed, the maximum principle applied to $u_{x}$ yields $u_{x}<0$ in $D_{T}$, implying $u>0$ in $D_{T}$ because of (3.5)).

The corresponding free boundary problem consists in finding a triple $T, s, u$ such that

(a) $T>0$,

(b) $s(t)$ is continuous and positive in $[0, T)$,

(c) $u(x, t)$ is continuous in $\bar{D}_{T}, u_{x}$ is continuous for $0 \leq x \leq s(t), 0<t<T, u_{x x}$ and $u_{t}$ are continuous in $D_{T}$,

(d) (3.1)-(3.6) are satisfied.

Existence and uniqueness of solutions of problem $(a)-(d)$ have been investigated in [20], [21], [14].

It is easily seen that if the function $g$ is regular enough $\left(g \in C^{2}, g^{\prime \prime}\right.$ Hölder-continuous at $x=1)$ and satisfies the compatibility conditions $g^{\prime}(0)=0, g(1)=g^{\prime}(1)=0, g^{\prime \prime}(1)=1$, then problem $(a)-(d)$ is reducible to a problem of type $(i)-(i v)$ of Sec. 2 for the function $z=u_{t}(x, t)$ and thus possesses a very smooth solution.

Here, we shall deal specifically with the case in which $g(x)$ represents the so-called equilibrium distribution [17]:

$$
g(x)=\frac{1}{2}(1-x)^{2} .
$$

Since $g^{\prime}(0) \neq 0$, the above argument does not apply, but we will show that the same result holds; we shall also get a sharp estimate of the time $T_{0}$ of total oxygen consumption. In a forthcoming paper, a much more general nonlinear problem of this kind will be analyzed.

Although uniqueness has been proved both in [20] and in [21], we report the following argument for its simplicity (see [29]).

THEOREM 3.1 (uniqueness). If $g(x)$ is nonnegative and nonincreasing $(g \not \equiv 0)$, problem $(a)-(d)$ has at most one solution. 
Proof. We have already noted that if $T, s, u$ is a solution, then $u>0, u_{x} \leq 0$ in $D_{T}$. Now, let $\left(T, s_{1}, u_{1}\right),\left(T, s_{2}, u_{2}\right)$ solve $(a)-(d)$ and set $W=u_{1}-u_{2}$. If $W \neq \equiv$, it must attain its (positive) maximum and/or its (negative) minimum somewhere on the curve $x=\min \left\{s_{1}(t), s_{2}(t)\right\}$. Let us suppose that $W$ is maximum at a point $\left(x_{0}, t_{0}\right)$ on this curve. Assuming $s_{1}\left(t_{0}\right) \leq s_{2}\left(t_{0}\right)$ contradicts $W\left(x_{0}, t_{0}\right)>0$ because $u_{1}\left(x_{0}, t_{0}\right)=0$ and $u_{2}\left(x_{0}, t_{0}\right) \geq 0$, while assuming $s_{1}\left(t_{0}\right)>s_{2}\left(t_{0}\right)$ contradicts $w_{x}\left(x_{0}, t_{0}\right)>0$ because $u_{1 x}\left(x_{0}, t_{0}\right) \leq 0$ and $u_{2 x}\left(x_{0}, t_{0}\right)=0$. Hence the proof of uniqueness.

The proof of the existence of a smooth solution to $(a)-(d)$ will be performed by means of a fixed point argument, which goes through the following steps ${ }^{2}$.

(1) Definition of the set $B(\widetilde{T}, X)$. Given two positive constants $\tilde{T}, X$, we detine the following set of functions $V:[0, \tilde{T}] \rightarrow \mathbb{R}$ :

$$
B(\tilde{T}, X)=\{V: V \in C[0, \tilde{T}],-X \leq V(t) \leq 0, t \in[0, \tilde{T}], V(0)=0\} .
$$

(2) Introduction of an auxiliary free boundary problem. Choose $b \in(0,1)$ arbitrarily and let $V \in B(\tilde{T}, X)$. Let us look for a solution $T, s, z$ of the following free boundary problem, which is similar to $(i)-(i v)$ of Sec. 2:

$$
\begin{aligned}
& z_{x x}-z_{t}=0 \quad b / 2<x<s(t), \quad 0<t<T \leq \tilde{T}, \\
& s(0)=1 \text {, } \\
& z(x, 0)=0, \quad b / 2<x<1 \text {, } \\
& z(b / 2, t)=V(t) \quad 0<t<T, \\
& z(s(t), t)=0, \quad 0<t<T, \\
& z_{x}(s(t), t)=-\dot{s}(t) \quad 0<t<T .
\end{aligned}
$$

Existence and uniqueness of the solution of (3.8)-(3.13) are ensured by the results of $[1, I]$ (see Secs. 6, 8).

Moreover,

$$
s \in C^{\infty}(0, T)
$$

(see [9], [25]), and two constants $T_{X} \in(0, \tilde{T}], A_{X}>0$ depending on $X$ (and $b$ ) can be found such that

$$
-A_{X} \leq \dot{s}(t) \leq 0, \quad \forall t \in\left[0, T_{X}\right]
$$

(see [1, I], Theorem 1, 2 for $\dot{s} \geq-A_{X}$, while $\dot{s} \leq 0$ follows from (3.12), (3.13) and $z \leq 0$ ).

(3) An auxiliary initial-boundary value problem. Let $\bar{T} \in\left(0, T_{X}\right]$ be such that

$$
s(t)>b, \quad t \in(0, \bar{T}),
$$

where $x=s(t)$ is the free boundary for (3.8)-(3.13).

\footnotetext{
${ }^{2}$ Different proofs for this existence theorem can be given; one of them will be found in the forthcoming paper announced above.
} 
Consider the following initial-boundary value problem:

$$
\begin{array}{cc}
v_{x x}-v=0, & \text { in } D_{T}, \\
v(x, 0)=-1, & 0<x<1, \\
v(0, t)=0, & 0<t<\bar{T}, \\
v_{x}(s(t), t)=0, & 0<t<\bar{T} .
\end{array}
$$

By the maximum principle we have $-1<v(x, t)<0, v_{x}(x, t)<0$ in $D_{T}$. Moreover, $v_{x}(b / 2, t)$ is continuous for $t \in[0, \bar{T}]$, hence $\left|v_{x}(b / 2, t)\right| \leq \bar{X}$ in $[0, \bar{T}]$ for some positive $\bar{X}$.

(4) Definition of the operator $\mathscr{T}$. Let

$$
V_{1}(t)=v_{x}(b / 2, t), \quad 0<t<\bar{T} .
$$

The equation

$$
V_{1}=\mathscr{T} V
$$

defines an operator

$$
\mathscr{T}: B(\tilde{T}, X) \rightarrow B(\bar{T}, \bar{X}) .
$$

(5) Solutions of $(a)-(d)$ associated with fixed points of $\mathscr{T}$. Suppose that the operator $\mathscr{T}$ has a fixed point $V$ in $B(\hat{T}, \hat{X})$ for some positive $\hat{T}, \hat{X}$; then the triple $\hat{T}, s$, $u$, with $s$ defined in (2) and

$$
u(x, t)=-\int_{x}^{s(t)}[\xi+v(\xi, t)] d \xi,
$$

where $v(x, t)$ is defined in (3), is a solution to $(a)-(d)$.

To prove this assertion we remark that under the above assumption we can identify $z$ with $v_{x}$ :

$$
z(x, t)=v_{x}(x, t), \quad b / 2 \leq x \leq s(t), \quad 0<t<T .
$$

Hence $v_{t}=z_{x}$ and from (3.13), (3.19)

$$
d v(s(t), t) / d t=v_{t}(s(t), t)=-\dot{s}(t),
$$

which in turn implies

$$
v(s(t), t)=-s(t) .
$$

On the basis of (3.23) and (3.24) it is easy to check that $\hat{T}, s, u$ solves $(a)-(d)$.

(6) For suitable $\hat{T}, \hat{X}$, the operator $\mathscr{T}$ maps $B(\hat{T}, \hat{X})$ into itself. First of all, let us find a time interval $\left(0, T^{\prime}\right)$ in which the function $s(t)$ issuing from (3.8)-(3.13) is greater than the given constant $b$ irrespective of the choice of $V$ in $B\left(T^{\prime}, X\right)$, for fixed $X$. We need the following equation:

$$
\left[s^{2}(t)-1\right] / 2=\int_{0}^{t} V(\tau) d \tau-\int_{b / 2}^{s(t)} x z(x, t) d x,
$$

resulting e.g. from formula $(3.5)$ of $[1, I]$. 
Since $z \leq 0$ and $V \geq-X$, the condition $1-2 X t \geq b^{2}$ will ensure $s(t) \geq b$. Thus we shall take

$$
T^{\prime}=\min \left\{(1-b)^{2} /(2 X), T_{X}\right\} .
$$

Now, if $t \in\left(0, T^{\prime}\right)$ it is true that $s(t)-b / 2>b / 2$ and by means of standard techniques (potential theory) we are able to determine a positive constant $\hat{X}$ (depending on $b$ only) such that

$$
-\hat{X} \leq V_{1}(t) \leq 0, \quad t \in\left(0, T^{\prime}\right)
$$

Therefore, if we define

$$
\hat{T}=\min \left\{\left(1-b^{2}\right) /(2 \hat{X}), T_{\hat{X}}\right\}
$$

we can assert that

$$
\mathscr{T}: B(\hat{T}, \hat{X}) \rightarrow B(\hat{T}, \hat{X})
$$

(7) $\mathscr{T}$ maps a closed, convex and compact subset of $B(\hat{T}, \hat{X})$ into itself. Let us introduce the Hölder norm

$$
\|V\|_{(0, T)}^{(\boldsymbol{x})}=\sup _{0<t^{\prime}<t<T}\left|V\left(t^{\prime}\right)-V\left(t^{\prime \prime}\right)\right| /\left(t^{\prime \prime}-t^{\prime}\right)^{\alpha},
$$

with $\alpha \in(0,1)$, and consider the following subset of $B(\hat{T}, \hat{X})$ :

$$
B_{x}(\hat{T}, \hat{X}, Y)=\left\{V: V \in B(\hat{T}, \hat{X}),\|V\|_{(0, \hat{r})}^{(\alpha)} \leq Y\right\},
$$

$Y$ being a positive constant.

As a set in $C[0, \hat{T}], B_{x}(\hat{T}, \hat{X}, Y)$ is closed, convex and compact.

By means of a standard use of potential theory a constant $\hat{Y}_{x}>0$ can be found, depending on $b$ only (for fixed $\alpha$ ), such that

$$
\left\|V_{1}\right\|_{(0, \hat{T})}^{(x)} \leq \hat{Y}_{x}
$$

irrespective of the choice of $V$ in $B(\hat{T}, \hat{X})$, i.e. $\mathscr{T}$ maps $B(\hat{T}, \hat{X})$ into $B_{x}(\hat{T}, \hat{X}, \hat{Y})$ and in particular

$$
\mathscr{T}: B_{\alpha}\left(\hat{T}, \hat{X}, \hat{Y}_{\alpha}\right) \rightarrow B_{\alpha}\left(\hat{T}, \hat{X}, \hat{Y}_{x}\right) .
$$

(8) Continuity of $\mathscr{T}$. Let us prove that

$$
\left\|\mathscr{T} V^{*}-\mathscr{T} V^{* *}\right\|_{C[0, \hat{T}]} \leq K\left\|V^{*}-V^{* *}\right\|_{C[0, \hat{T}]}, \forall V^{*}, V^{* *} \in B(\hat{T}, \hat{X}),
$$

where $\|\cdot\|_{C[0, \hat{T}]}$ denotes the sup in $(0, \hat{T})$ and the constant $K$ depends on $b$ only.

We denote by $s^{*}, s^{* *}$ the free boundaries of problem (3.8)-(3.13) with the respective functions $V^{*}, V^{* *}$ given in the boundary condition (3.10). The inequality

$$
\left\|\mathscr{T} V^{*}-\mathscr{T} V^{* *}\right\|_{C[0, \hat{T}]} \leq K_{1}\left\|s^{*}-s^{* *}\right\|_{C[0, \hat{T}]}
$$

with $K_{1}$ depending on $b$ (also through $\hat{X}$ and the constant $A_{\hat{X}}$ appearing in (3.15)), can be proved as follows.

Consider the solution $v$ of (3.16)-(3.19) with a boundary $x=s(t)$ satisfying (3.15). We can write

$$
v=-\operatorname{erf}(x / 2 \sqrt{ } t)+v_{0}
$$


where $v_{0}$ solves the heat equation in $D_{T}$ with the initial condition $v_{0}(x, 0)=0$ and the boundary conditions $v_{0}(0, t)=0$ and $v_{0, x}(s(t), t)=\exp \left[-s^{2}(t) /(4 t)\right] /(\pi t)^{1 / 2}$.

The function $\tilde{v}_{0}=v_{0, x}$ satisfies

$$
\begin{aligned}
\tilde{v}_{0, x x}-\tilde{v}_{0, t} & =0 \quad \text { in } \quad D_{\bar{T}}, \\
\tilde{v}_{0}(x, 0) & =0, \quad 0<x<1, \\
\tilde{v}_{0, x}(0, t) & =0, \quad 0<t<\bar{T}, \\
\tilde{v}_{0}(s(t), t) & =\exp \left[-s^{2}(t) /(4 t)\right] /(\pi t)^{1 / 2}, \quad 0<t<\bar{T} .
\end{aligned}
$$

Denoting by $v^{*}, \tilde{v}_{0}^{*}, v^{* *}, \tilde{v}_{0}^{* *}$ the respective functions obtained replacing $s$ by $s^{*}$ or $s^{* *}$, it is true that

$$
\mathscr{T} V^{*}-\mathscr{T} V^{* *}=\tilde{v}_{0}^{*}(b / 2, t)-\tilde{v}_{0}^{* *}(b / 2, t)
$$

The estimate

$$
\left\|\tilde{v}_{0}^{*}(b / 2, t)-\tilde{v}_{0}^{* *}(b / 2, t)\right\|_{C[0, \hat{T}]} \leq K_{1}\left\|s^{*}-s^{* *}\right\|_{C[0, \hat{\tau}]},
$$

implying (3.34) is obtained by applying results in [26] (Corollary 3.3) and in [27] (Theorem 4).

Now we claim that

$$
\left\|s^{*}-s^{* *}\right\|_{C[0, \uparrow]} \leq K_{2}\left\|V^{*}-V^{* *}\right\|_{C[0, \uparrow]},
$$

with $K_{2}$ dependent on $b$. This is an immediate consequence of Theorem 5 of $[1, I]$ : the constant $K_{2}$ depends on $b$ and on $\hat{X}, A_{\hat{X}}$, in turn depending on $b$.

Combining (3.34) and (3.35) yields (3.33)

(9) Existence of a fixed point of $\mathscr{T}$ in $B(\hat{T}, \hat{X})$. Owing to (7) and (8) we can use Schauder's theorem, concluding that $\mathscr{T}$ has at least one fixed point in $B_{\alpha}(\hat{T}, \hat{X}$, $\left.\hat{Y}_{x}\right) \subset B(\hat{T}, \hat{X})$.

Finally, from (9) and (5) we infer the existence of a solution $\hat{T}, s, u$ to $(a)-(d)$. The free boundary $x=s(t)$ of this solution can be identified with the free boundary of problem $(3.8)-(3.19)$ and therefore it is analytic in $(0, \hat{T})[9]$. Thus we have proved.

Theorem 3.2. Problem $(a)-(d)$ with $g(x)$ specified by (3.7) possesses a solution $T, s, u$, whose free boundary is analytic in $(0, \hat{T})$.

Remark 3.3. By setting $z=u_{t}$, problem $(a)-(d)$ can be reformulated in the form (i)-(iv) of Section 2, giving the "initial" condition $z(x, \varepsilon)=u_{t}(x, \varepsilon)$. Actually, $z$ is the function solving (3.8)-(3.13). It is easily seen that

$$
\int_{0}^{s(t)} z(x, t) d x=-s(t), \quad \forall t \in(0, \hat{T})
$$

therefore the associated problem of type (i)-(iv) has $Q=0$ (the limit of $z$ for $t \rightarrow 0$ is thus a " $\delta$-function"). Moreover, $u_{t x}(x, \varepsilon)$ is positive (note that $u_{t x}(0, t)=0, u_{t}(s(t), t)=0$ and

\footnotetext{
${ }^{3}$ More precisely, Theorem 5 of $[1, I]$ shows that the constant $K_{2}$ in (3.35), and consequently the constant $K$ in (3.33), is proportional to $\hat{T}$. Therefore taking $\hat{T}$ sufficiently small we deduce that $\mathscr{T}$ is contractive.
} 
$u_{t x}(x, 0)=0$ out of the origin, and that

$$
\left.\limsup _{(x, t) \rightarrow(0,0)} u_{t x}(x, t)=+\infty, \quad \liminf _{(x, t) \rightarrow(0,0)} u_{t x}(x, t)=0\right) .
$$

Consequently, Theorem 2.9 ensures that the problem under consideration is of type (B). Moreover, (2.35), (2.36) and (2.37) of Theorem 2.12 apply in the following sense.

Noting that $\lim _{t \rightarrow 0} \int_{0}^{s(t)} x z(x, t) d x=0$, we can take $M_{1}=0$ in (2.36) and get the estimate $T_{0}<1 / 2$, which can also be found e.g. in [14].

Considering next $\int_{0}^{s(t)} x^{2} z(x, t) d x=\int_{0}^{s(t)} x^{2}\left(u_{x x}-1\right) d x$, we see that it vanishes as $t \rightarrow 0$ : this allows us to put $M_{2}=0$ in (2.35), getting

$$
\int_{0}^{T_{0}} s(t) d t=\frac{1}{6}
$$

The estimate $T_{0}<1 / 2$ obtained in Remark 3.3 is rather crude. We recall that in [17] the value $T_{0}=\pi / 16$ was assumed as a good approximation. As a matter of fact it can be shown that $\pi / 16$ is a lower bound for $T_{0}$, but quite close to the actual value. This is the aim of Propositions 3.4 and 3.5 below.

Proposition 3.4. The following estimate holds in $D_{T_{0}}$ :

$$
u(x, t) \geq(x-1)^{2} / 2-2(t / \pi)^{1 / 2} \exp \left[-x^{2} /(4 t)\right]+x \operatorname{erfc}[x /(2 \sqrt{ } t)] .
$$

Proof. From the Green's formula we have immediately:

$$
\begin{aligned}
v(x, t)= & -\int_{0}^{1} G(x, t ; \xi, 0) d \xi+\int_{0}^{t} s(\tau) G_{\xi}(x, t ; s(\tau), \tau) d \tau \\
& -\int_{0}^{t} s(\tau) G(x, t ; s(\tau), \tau) \dot{s}(\tau) d \tau
\end{aligned}
$$

where we use the standard notation:

$$
\begin{aligned}
G(x, t ; \xi, \tau) & =\Gamma(x, t ; \xi, \tau)-\Gamma(-x, t ; \xi, \tau), \\
N(x, t ; \xi, \tau) & =\Gamma(x, t ; \xi, \tau)+\Gamma(-x, t ; \xi, \tau), \\
\Gamma(x, t ; \xi, \tau) & =[4 \pi(t-\tau)]^{-1 / 2} \exp \left[-(x-\xi)^{2} / 4(t-\tau)\right] .
\end{aligned}
$$

Differentiating (3.38) with respect to $x$, we get after simple calculations:

$$
v_{x}(x, t)=-N(x, t ; 0,0)-\int_{0}^{t} \dot{s}(\tau) N(x, t ; s(\tau), \tau) d \tau .
$$

Since $\dot{s} \leq 0$ and $N>0$ we obtain

$$
v_{x}(x, t) \geq-N(x, t ; 0,0) .
$$

But (3.39) is a lower estimate for $u_{t}(x, t)$ since

$$
v(x, t)=u_{x}(x, t)-x
$$

as is easily verified. Hence

$$
u(x, t) \geq \frac{1}{2}(x-1)^{2}-\int_{0}^{t}(\pi \tau)^{-1 / 2} \exp \left[-x^{2} / 4 \tau\right] d \tau .
$$


By performing the integration in (3.40), (3.37) is obtained.

Now we prove

Proposition 3.5. The following estimate holds in $D_{T_{0}}$ :

$$
\begin{gathered}
u(x, t) \leq 2 \sum_{n=0}^{\infty} \exp \left(-a_{n}^{2} t\right) \cos a_{n} x \cdot a_{n}^{-2}\left\{1-(-1)^{n} a_{n}^{-1}\right\} \\
-\sum_{n=-\infty}^{+\infty}(-1)^{n} \pi^{-1 / 2} \int_{0}^{t}\left\{\operatorname{erf}\left[\frac{1}{2}(x-2 n+1-16 \tau / \pi) /(t-\tau)^{1 / 2}\right]\right. \\
\left.-\operatorname{erf}\left[\frac{1}{2}(x-2 n-1+16 \tau / \pi) /(t-\tau) \frac{1}{2}\right]\right\} d \tau,
\end{gathered}
$$

with $a_{n}=(2 n+1) \pi / 2$.

Proof. The right-hand side of (3.41) is the solution of the following problem:

$$
\begin{aligned}
U_{x x}-U_{t} & =q(x, t), & & 0<x<1, t>0, \\
U(x, 0) & =(1-x)^{2} / 2, & & 0<x<1, \\
U_{x}(0, t) & =0, & & t>0, \\
U(1,0) & =0, & & t>0,
\end{aligned}
$$

where $q(x, t)$ is the characteristic function of the triangle bounded by the straight lines $x=0, t=0$ and $x+16 t / \pi=1$. Now we remark that (3.40) provides the following lower estimate of the time of total oxygen consumption:

$$
T_{0}>\pi / 16
$$

and that the free boundary lies on the right of the segment joining the points $(1,0)$ and $(0, \pi / 16) .{ }^{4}$ Thus an easy application of the maximum principle leads to $(3.41)$.

4. Some comparison theorems; a nonexistence theorem for the supercooled liquid problem. Let us replace the boundary condition (3.4) with

$$
u_{x}(0, t)=-Q,
$$

with $Q=$ constant.

As a preliminary, we study the sign of the function $u(x, t)$ in $D_{T}$, assuming $T, s, u$ to be a solution to $(a)-(d)$ with the above substitution.

As in Sec. 3, we assume that $g$ is continuously differentiable in $[0,1]$. Here we assume also the compatibility conditions

$$
g(1)=0, \quad g^{\prime}(1)=0, \quad g^{\prime}(0)=-Q .
$$

We shall consider the two cases $Q \geq 0$ and $Q<0$ separately, adding some special assumption on $g$.

Proposition 4.1. If $Q \geq 0$ and $g^{\prime}(x) \leq 0(g \neq 0$ if $Q=0)$, it is true that $u(x, t)>0$ in $D_{T}$.

Proof. The proof is elementary, since the above assumptions imply $u_{x} \leq 0$ in $\bar{D}_{T}$.

\footnotetext{
${ }^{4}$ The free boundary for problem (3.1)-(3.6) with the initial condition (3.7) is such that $\ddot{s}<0$. This has been proved by the present authors in the paper Convexity of the free boundary in some classical free boundary problems, to appear on Riv. Mat. Univ. Parma.
} 
Proposition 4.2. If $Q<0$ suppose that the derivative $g^{\prime \prime}(x)$ is piecewise continuous and bounded in $[0,1]$ and that $g(x)$ is nonnegative. We consider two cases:

$(\alpha)$ if

$$
h(x) \equiv g^{\prime \prime}(x)-1 \geq 0 \quad \text { in } \quad(0,1)
$$

it is true that $u(x, t)>0$ in $D_{T}$;

$(\beta)$ if

$$
h(x) \leq 0, \quad \bar{h} \equiv \min _{x \in[0,1]} h(x)<0,
$$

suppose that $g^{\prime}(x)$ changes its sign only once in $(0,1)$ and that

$$
g(x)>0, \quad 0 \leq x<1
$$

then $u(x, t)>0$ in $D_{t^{*}}$, where

$$
t^{*}=g(0) /|\bar{h}|
$$

Proof. Case $(\alpha)$. Consider a solution $T, s, u$ of $(a)-(d)$. If (4.3) is satisfied the function $z=u_{t}$ solves the system (2.1), (2.3), (2.4), (2.5), with $h$ defined as in (4.3). Then $u_{t} \geq 0^{5}$ in $D_{T}$ and this implies $u>0$ in $D_{T}$, because of $g \geq 0$.

Case $(\beta)$. If (4.4) holds true we can assert that

$$
\bar{h}<u_{t}<0 \text { in } D_{T}
$$

by virtue of the maximum principle. Therefore, if $t_{0}$ is the first instant in which $u(0, t)$ vanishes (recall (4.5)), it is

$$
t_{0} \geq t^{*}
$$

with $t^{*}$ defined in (4.6).

Since $T, s, z,\left(z=u_{t}\right)$, solves (i)-(iv) of Sec. 2 , owing to (4.7) it is true that $\dot{s} \leq 0$ and from (4.7) we have

$$
u(x, t)>0 \text { for } s(T)<x<s(t), \quad 0<t<T .
$$

This prevents a level curve $u=0$ from originating from $t=0$.

Thus, owing to (4.5), either the first time $u(x, t)$ vanishes out of the free boundary is $t_{0}$ (then the Proposition is proved because of (4.8)), or there exists a time $t_{1}<t_{0}$ such that

$$
u(x, t)>0 \text { for } 0<x<s(t), \quad 0<t<t_{1}
$$

and that for some $x_{0} \in\left(0, s\left(t_{1}\right)\right)$

$$
u\left(x, t_{1}\right)>0, \quad x \neq x_{0}, \quad u\left(x_{0}, t_{1}\right)=0 .
$$

In such a case $u_{x}\left(x_{0}, t_{1}\right)=0$. Moreover, being $u\left(s\left(t_{1}\right), t_{1}\right)=0$, at least one point $x_{1} \in\left(x_{0}, s\left(t_{1}\right)\right)$ would exist in which $u_{x}\left(x_{1}, t_{1}\right)=0$. However, the assumption that $g^{\prime}$ changes its sign only once in $(0,1)$ implies that not more than one level curve $u_{x}=0$ can exist in $D_{T}$ and we have a contradiction.

\footnotetext{
${ }^{5} u_{t}=0$ only when $g^{\prime \prime}=1$, which corresponds to the equilibrium solution.
} 
We are now able to prove a monotone dependence theorem for the oxygen diffusionconsumption problem. Let $T, s_{1}, u_{1} ; T, s_{2}, u_{2}$ be solutions of the respective problems

$$
\begin{aligned}
u_{i, x x}-u_{i, t} & =1 & & \text { in } \quad D_{i, T}=\left\{(x, t): 0<x<s_{i}(t), 0<t<T\right\}, \\
s_{i}(0) & =b_{i}>0, & & \\
u_{i}(x, 0) & =g_{i}(x), & & 0<x<b_{i}, \\
u_{i, x}(0, t) & =-Q_{i}, & & 0<t<T, \\
u_{i}\left(s_{i}(t), t\right) & =0, & & 0<t<T, \\
u_{i, x}\left(s_{i}(t), t\right) & =0, & & 0<t<T, \quad i=1,2 .
\end{aligned}
$$

We state the following theorem.

THEOREM 4.3. Let $Q_{2}, g_{2}$ satisfy the assumptions of Proposition 4.1; then

$$
\begin{aligned}
b_{1}<b_{2}, \quad g_{1}(x) & \leq g_{2}(x) \text { for } 0<x<b_{1}, \\
Q_{1} & \leq Q_{2} \Rightarrow s_{1}(t)<s_{2}(t), \quad 0<t<T .
\end{aligned}
$$

If $Q_{2}, g_{2}$ satisfy the assumptions of Proposition 4.2, then the implication (4.10) remains true in the case $(\alpha)$, while in the case $(\beta)$ it is valid for $0<t<t^{*}$.

Proof. First of all, we remark that the condition $s(0)=b>0$ can always be reduced to $s(0)=1$ by rescaling the space and time variables; thus the results obtained so far are independent of the value of $s(0)$. In particular, we are allowed to say that $u_{2}(x, t)>0$ in $D_{T}$, if $Q_{2}, g_{2}$ satisfy the assumptions of Proposition 4.1 or of Proposition 4.2, case $(\alpha)$, and that $u_{2}(x, t)>0$ in $D_{t^{*}}$ if $Q_{2}, g_{2}$ are assumed to be as in Proposition 4.2, case $(\beta)$.

Suppose now that the curves $x=s_{1}(t), x=s_{2}(t)$ meet before $u_{2}$ changes its sign and let $\bar{t}$ be such that

$$
s_{1}(\bar{t})=s_{2}(\bar{t}), \quad s_{1}(t)<s_{2}(t) \quad \text { for } \quad 0<t<\bar{t} .
$$

In the domain $D_{1, i}$ the difference

$$
W=u_{2}-u_{1}
$$

satisfies the heat equation and the conditions

$$
\begin{aligned}
W(x, 0) & =g_{2}(x)-g_{1}(x) \geq 0, & & 0<x<b_{1}, \\
W_{x}(0, t) & =Q_{1}-Q_{2} \leq 0, & & 0<t<\bar{t}, \\
W\left(s_{1}(t), t\right) & =u_{2}\left(s_{1}(t), t\right)>0, & & 0<t<\bar{t} ;
\end{aligned}
$$

therefore $W\left(s_{1}(\bar{t}), \bar{t}\right)=0$ is a minimum. Hence $W_{x}\left(s_{1}(\bar{t}), \bar{t}\right)<0$ and we have a contradiction to $u_{1, x}\left(s_{1}(\bar{t}), \bar{t}\right)=u_{2, x}\left(s_{2}(\bar{t}), \bar{t}\right)=0$.

We conclude that $s_{1}(t)<s_{2}(t)$ until $u_{2}(x, t)$ is positive. This proves the theorem.

Recalling now that problem $(a)-(d)$, with $(4.1)$ in place of (3.4), is equivalent to problem (i)-(iv), we can obtain a comparison theorem for (i)-(iv).

More precisely, let $T, s, z$ be a solution of (i)-(iv) with $s(0)=b$ and define

$$
Q=b+\int_{0}^{b} h(x) d x
$$


Then the triple $T, s, u$ with

$$
u(x, t)=[x-s(t)]^{2} / 2+\int_{x}^{s(t)} d \xi \int_{\xi}^{s(t)} z(\eta, t) d \eta
$$

solves $(a)-(d)$ with the boundary condition (4.1) and the initial datum

Obviously $h=g^{\prime \prime}-1$.

$$
g(x)=(x-b)^{2} / 2+\int_{x}^{b} d \xi \int_{\xi}^{b} h(\eta) d \eta .
$$

If $T, s_{i}, z_{i}, i=1,2$, are two solutions of (i)-(iv) corresponding to initial data $s_{i}(0)=b_{i}, z_{i}(x, 0)=h_{i}(x), i=1,2$, from Theorem 4.3 we deduce

Corollary 4.4. The implication

$$
\begin{gathered}
b_{1}<b_{2}, \quad\left(x-b_{1}\right)^{2} / 2+\int_{x}^{b_{1}} d \xi \int_{\xi}^{b_{1}} h_{1}(\eta) d \eta \leq\left(x-b_{2}\right)^{2} / 2 \\
\quad+\int_{x}^{b_{2}} d \xi \int_{\xi}^{b_{2}} h_{2}(\eta) d \eta \text { for } 0<x<b_{1}, \\
Q_{1} \leq Q_{2} \Rightarrow s_{1}(t)<s_{2}(t)
\end{gathered}
$$

is valid in the same sense as Theorem 4.3, when $Q_{2}$ and $g_{2}$ from (4.11) and (4.13) satisfy the assumption stated there.

We are now in position to improve Theorem 2.2.

THEOREM 4.5. If $h(x)<-1$ in $(0,1)$, problem (i)-(iv) has no solution.

Proof. Let us assume that $T, s, z$ is a solution of problem (i)-(iv) with a given $h<-1$ and compare it with the solutions $T_{n}, s_{n}, z_{n}, n=1,2$, of (i)-(iv) corresponding to initial data $s_{n}(0)=1+1 / n, z_{n}(x, 0)=h_{n}(x), 0<x<b$. We define $h_{n}$ as follows

Note that

$$
\begin{aligned}
& h_{n}(x)=0 \text { for } 1 \leq x \leq 1+1 / n, \\
& h_{n}(x)=-1-1 / n \text { for } 0 \leq x<1 .^{6}
\end{aligned}
$$

$$
Q_{n}=1+\frac{1}{n}+\int_{0}^{1+1 / n} h_{n}(x) d x=0,
$$

and that $h_{n}$ is nondecreasing. Therefore the corresponding solutions are of type (B) (Theorem 2.9), with a time of existence $T_{0}^{(n)}$ which can be estimated as follows

$$
T_{0}^{(n)}<1 / 2 n+1 / 2 n^{2}
$$

(use the same argument as in the proof of (2.36)).

According to (4.13),

$$
\begin{aligned}
& g_{n}(x)=\left[2(1-x)-\left(1-x^{2}\right)^{2}+1 / n\right] /(2 n), \quad 0 \leq x \leq 1, \\
& g_{n}(x)=(1-x+1 / n)^{2} / 2, \quad 1<x<1+1 / n
\end{aligned}
$$

and Proposition 4.1 applies to the associated triples $T_{0}^{(n)}, s_{n}, u_{n}$.

\footnotetext{
${ }^{6}$ Of course, (iii) has to be slightly modified in order to include piecewise continuous initial data.
} 
For $n$ sufficiently large, $h(x)<h_{n}(x), 0<x<1$, and the comparison between $s$ and $s_{n}$ can be made by exploiting Corollary 4.4.

We infer that $T, s, z$ must be of type (B) or (C) with a total time of existence not exceeding $T_{0}^{(n)}$.

By letting $n \rightarrow \infty$, we see that $T_{0}^{(n)} \rightarrow 0$; this contradicts the assumption that a solution $T, s, z$ exists.

Appendix. An extension of Theorem 2.10. For $h(x) \leq 0, x \in(0,1)$, and $1 \geq Q \geq 0$, the condition for (C) may be generalized to: if $f(x) \in C^{2}[0,1]$ satisfies $f^{\prime}(0) \geq 0, f^{\prime \prime}(x) \geq 0$, $x \in(0,1)$, then

$$
\int_{Q}^{1} f(x) d x+\int_{0}^{1} f(x) h(x) d x<0 \Rightarrow(\mathrm{C}) .
$$

Proof. Define $F(t)=\int_{0}^{s(t)} f(x)(1+z(x, t)) d x$. Then, assuming $z$ and $s$ are well enough behaved to permit differentiation under the integral sign,

$$
\begin{aligned}
\dot{F} & =f(s) \dot{s}+\int_{0}^{s} f(x) z_{t}(x, t) d x \\
& =f^{\prime}(0) z(0, t)+\int_{0}^{s} f^{\prime \prime}(x) z(x, t) d x,
\end{aligned}
$$

using (i)-(iv). But $z(x, t) \leq 0$, so $\dot{F} \leq 0$. Now if (A) or (B) holds, $s \rightarrow Q$ as $t \rightarrow \infty$ or as $t \rightarrow T_{0}$. Also, as long as the solution exists, $s+\int_{0}^{s} z(x, t) d x=Q$ and so, assuming $z$ is well behaved, either $s \rightarrow 0$ or $z \rightarrow 0$ as $t \rightarrow \infty$ or $t \rightarrow T_{0}$. Thus, in either (A) or (B), $F \rightarrow \int_{0}^{Q} f(x) d x$ as $s \rightarrow Q$. Applying $\dot{F} \leq 0$, we obtain

$$
\int_{0}^{Q} f(x) d x \leq F(0)=\int_{0}^{1} f(x) d x+\int_{0}^{1} f(x) h(x) d x .
$$

Thus, $\int_{Q}^{1} f(x) d x+\int_{0}^{1} f(x) h(x) d x<0 \Rightarrow(\mathrm{C})$.

Putting $f=x, x^{2}$ yields $\left(2.24^{\prime}\right)$ but, as we shall shortly show, in an oxygen diffusion problem of the type considered in Sec. 3, the conditions of $\left(2.24^{\prime}\right)$ are not necessary for $(C)$.

It is convenient to modify the above result before applying it to oxygen diffusion problems. We must of course only consider situations where $g^{\prime \prime}(1)=1$ and the compatability conditions (4.2) are satisfied so that the supercooled Stefan problem is related to the oxygen diffusion problem through $z=u_{t}$. In such a case $h=g^{\prime \prime}-1$ and we now show that the existence of $f \in C^{2}[0,1]$ such that $f^{\prime}(0) \geq 0, f^{\prime \prime}(x) \geq 0, x \in(0,1)$ and $\int_{Q}^{1} f(x) d x+\int_{0}^{1} f(x) h(x) d x<0$ is a necessary and sufficient condition for the existence of an $x_{-} \in(0,1)$ such that $g\left(x_{-}\right)<0$. Then the existence of such an $x_{-}$will imply (C).

(i) Sufficiency. Substitution for $h$ gives, trivially,

$$
\int_{Q}^{1} f(x) d x+\int_{0}^{1} f(x) h(x) d x<0 \Leftrightarrow \int_{0}^{Q} f(x) d x>\int_{0}^{1} f^{\prime \prime}(x) g(x) d x+Q f(0)+f^{\prime}(0) g(0) .
$$

Hence, if we assume $g(x)$ is always positive,

$$
\int_{0}^{Q} f(x) d x>\int_{0}^{Q} f^{\prime \prime}(x) g(x) d x+Q f(0)+f^{\prime}(0) g(0) .
$$


But $h(x) \leq 0 \Rightarrow g^{\prime \prime}(x) \leq 1$ and $g^{\prime}(Q) \leq 0$. Hence, since by assumption $g(Q) \geq 0$, we may conclude $g(x) \geq \frac{1}{2}(x-Q)^{2}, x \in[0, Q]$. Finally, substituting for $g$ and integrating by parts yields

$$
\int_{0}^{Q} f(x) d x>\int_{0}^{Q} \frac{1}{2}(x-Q)^{2} f^{\prime \prime}(x) d x+Q f(0)+\frac{1}{2} Q^{2} f^{\prime}(0)=\int_{0}^{Q} f(x) d x
$$

and we have a contradiction.

(ii) Necessity. If $g\left(x_{-}\right)<0$ then, since $g$ is continuous, there is a number $a \in\left(x_{-}, 1\right)$ such that $g(x)<0$ for $x \in\left(x_{-}, a\right)$. Now choose $f$ so that $f(0)=f^{\prime}(0)=0$ with $f^{\prime \prime}(x)=0$ for $x \leq x_{-}$and $x \geq a$, and with $f^{\prime \prime}(x)>0$ for $x \in\left(x_{-}, a\right)$.

For such an $f$,

$$
\int_{0}^{Q} f(x) d x \geq 0>\int_{0}^{1} f^{\prime \prime}(x) g(x) d x+Q f(0)+f^{\prime}(0) g(0) .
$$

Note: if $g\left(x_{-}\right)<0, u\left(x_{-}, t\right)<0$ as long as the solution exists so that $s(t)>x_{-}$.

Example. We conclude by exhibiting a function $g(x)$ for the oxygen diffusion problem such that $(\mathrm{C})$ occurs in the related supercooled Stefan problem and yet the conditions of $\left(2.24^{\prime}\right)$ are not satisfied. Just considering the case $Q=0$, this means we require $g(0)>0$ and $\int_{0}^{1} g(x) d x>0$. Moreover, we require $g^{\prime \prime}(x) \leq 1$ for $x \in[0,1]$ and $g^{\prime \prime}(1)=1$ in addition to the compatability conditions (4.2). The function

$$
g(x)= \begin{cases}\left(3 / 25 \pi^{2}\right) \cos (5 \pi x / 2) & 0 \leq x \leq \frac{4}{5}-\varepsilon \\ \left(3 / 25 \pi^{2}\right) \cos ^{2}(5 \pi x / 2), & \frac{4}{5}+\varepsilon \leq x \leq 1,\end{cases}
$$

with $g$ not too large and $g$ continuous in $\left[\frac{4}{5}-\varepsilon, \frac{4}{5}+\varepsilon\right]$, meets all these requirements and is negative in $\left(\frac{1}{5}, \frac{3}{5}\right)$. Hence we may conclude that $(C)$ occurs.

\section{REFERENCES}

[1] A. Fasano and M. Primicerio, General free boundary problems for the heat equation I, J. Math. Anal. Appl. 57, 694-723 (1977); II, ibid. 58, 202-231 (1977); III, ibid. 59, 1-14 (1977)

[2] A. Schatz, Free boundary problems of Stefan type with prescribed flux, J. Math. Anal. Appl. 28, 569-580 (1969)

[3] B. Sherman, A general one-phase Stefan problem, Quart. Appl. Math. 28, 377-382 (1970)

[4] B. Sherman, General one-phase Stefan problems and free boundary problems for the heat equation with Cauchy data prescribed on the free boundary, SIAM J. Appl. Math. 20, 555-570 (1971)

[5] J. Bather, Optimal stopping problems for Brownian motion, Adv. Appl. Prob. 2, 259-286 (1970)

[6] A. Bensoussan and J. L. Lions, Problèmes de temps d'arrêt optimal et inéquations variationelles paraboliques, Applic. Anal. 3, 267-295 (1973)

[7] D. B. Kotlow, A free boundary problem connected with the optimal stopping problem for diffusion processes, Trans. Amer. Math. Soc. 194, 457-478 (1973)

[8] A. Friedman, Parabolic variational inequalities in one space dimension and smoothness of the free boundary, J. Funct. Anal. 18, 151-176 (1975)

[9] A. Friedman, Analyticity of the free boundary for the Stefan problem, Arch. Rat. Mech. Anal. 61, 97-125 (1976)

[10] A. Friedman and R. Jensen, Convexity of the free boundary in the Stefan problem and in the dam problem, Arch. Rat. Mech. Anal. 67, 1-24 (1977)

[11] R. Jensen, Smoothness of the free boundary in the Stefan problem with supercooled water, to appear

[12] P. van Moerbecke, An optimal stopping problem for linear reward, Acta Math. 132, 1-41 (1974)

[13] P. van Moerbecke, About optimal stopping problems, Arch. Rat. Mech. Anal. 60, 101-148 (1976) 
[14] E. Magenes, Topics in parabolic equations; some typical free boundary problems, in Boundary value problems for linear evolution partial equations (ed. Garnir), 1977, 239-312

[15] J. R. Ockendon and W. R. Hodgkins, Moving boundary problems in heat flow and diffusion, Clarendon Press, Oxford (1975)

[16] D. G. Wilson, A. D. Solomon and P. T. Boggs, Moving boundary problems, Academic Press (1978)

[17] J. Crank and R. S. Gupta, A moving boundary problem arising from the diffusion of oxygen in absorbing tissues, J. Inst. Maths. Applics. 10, 19-33 (1972)

[18] E. Hansen and P. Hougaard, On a moving boundary problem from biomechanics, J. Inst. Maths. Applics. 13, 385-398 (1974)

[19] C. Baiocchi and G. A. Pozzi, An evolution variational inequality related to a diffusion-absorption problem, Appl. Math. Optim. 2, 304-314 (1976)

[20] C. Baiocchi and G. A. Pozzi, Error estimates and free boundary convergence for a finite difference discretization of a parabolic variational inequality, to appear RAIRO.

[21] J. C. W. Rogers, A free boundary problem as diffusion with nonlinear absorption, J. Inst. Maths. Applics. 20, 261-268 (1977)

[22] A. E. Berger, M. Ciment and J. C. W. Rogers, Numerical solution of a diffusion consumption problem with a free boundary, SIAM J. Num. Anal. 12, 646-672 (1975)

[23] A. E. Berger, The truncation method for the solution of a class of variational inequalities, RAIRO 10, 29-42 (1976)

[24] H. Block, Sur les équations aux dérivees partielles de type parabolique, Ark. Matem. Astr. Fys. 6, n. 31 (1910)

[25] D. G. Schaeffer, A new proof of the infinite differentiability of the free boundary in the Stefan problem, J. Diff. Eq. 20, 266-269 (1976)

[26] A. Fasano and M. Primicerio, La diffusione del calore in uno strato di spessore variabile in presenza di scambi termici non lineari con lambiente, Rend. Sem. Mat. Univ. Padova 50, 269-330 (1973)

[27] A. Fasano and M. Primicerio, Su un problema unidimensionale di diffusione in un mezzo a contorno mobile con condizioni ai limiti non lineari, Ann. Mat. Pura Appl. (IV) 93, 333-357 (1972)

[28] Carslaw and Jaeger, Conduction of heat in solids, Clarendon Press, Oxford (1959)

[29] S. N. Kruzkov, On some problems with unknown boundaries for the heat conduction equation, Prikl. Mat. Meh. 31, 1009-1020 (1967) 